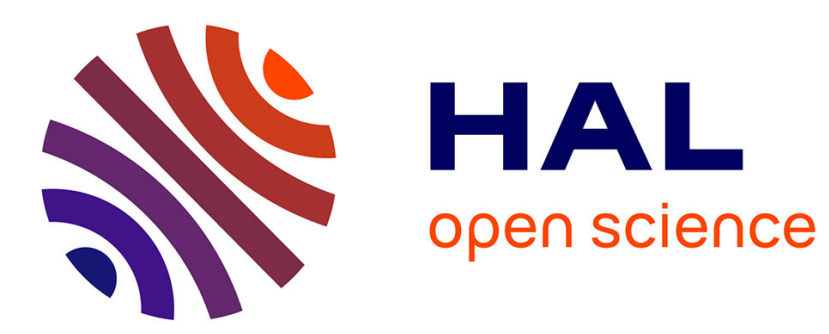

\title{
Polarization Spectroscopy of an Isolated Quantum Dot and an Isolated Quantum Wire
}

\author{
V. Kats, A.V. Platonov, G. E Tsyrlin, A. D Buravlev, A. Delga, L. Besombes, \\ H. Mariette, V. Kochereshko
}

\section{- To cite this version:}

V. Kats, A.V. Platonov, G. E Tsyrlin, A. D Buravlev, A. Delga, et al.. Polarization Spectroscopy of an Isolated Quantum Dot and an Isolated Quantum Wire. Physics of the Solid State, 2018, 60 (12), pp.2623-2627. 10.1134/S1063783418120156 . hal-02058731

\section{HAL Id: hal-02058731 https://hal.science/hal-02058731}

Submitted on 20 Mar 2019

HAL is a multi-disciplinary open access archive for the deposit and dissemination of scientific research documents, whether they are published or not. The documents may come from teaching and research institutions in France or abroad, or from public or private research centers.
L'archive ouverte pluridisciplinaire HAL, est destinée au dépôt et à la diffusion de documents scientifiques de niveau recherche, publiés ou non, émanant des établissements d'enseignement et de recherche français ou étrangers, des laboratoires publics ou privés. 


\title{
Polarization Spectroscopy of an Isolated Quantum Dot and an Isolated Quantum Wire
}

\author{
V. N. Kats ${ }^{a}$ * , A. V. Platonov ${ }^{a}$, G. E. Tsyrlin ${ }^{a}$, A. D. Buravlev ${ }^{a}$, A. Delga $^{b}$, L. Besombes ${ }^{b}$, \\ H. Mariette ${ }^{b}$, and V. P. Kochereshko ${ }^{a}$ \\ ${ }^{a}$ Ioffe Institute, St. Petersburg, 194021 Russia \\ ${ }^{b}$ Institut Néel, 38042 Grenoble, France \\ *e-mail: Vladimir.N.Kats@gmail.com
}

Received May 22, 2018

\begin{abstract}
Photoluminescence spectra of an isolated GaAs quantum dot within an AlGaAs quantum wire are studied. The examination of behavior of spectra in a magnetic field provided an opportunity to estimate the exciton binding energy in the quantum dot, which turned out to be 10 times higher than the bulk exciton binding energy in GaAs. It is demonstrated that the signal of exciton photoluminescence from the quantum dot emitted along the nanowire axis is linearly polarized. At the same time, the photoluminescence signal propagating in the direction perpendicular to the nanowire axis is almost unpolarized. This may be attributed to the nonaxial dot positioning in the wire under a giant increase in the binding energy of an exciton affected by an image potential.
\end{abstract}

DOI: $10.1134 / \mathrm{S} 1063783418120156$

\section{INTRODUCTION}

Molecular-beam epitaxy (MBE) with a catalyst in the form of metallic droplets on the surface is an advanced technique developed in recent years that allows one to synthesize quantum wires (nanowires, NWs) up to $10 \mu \mathrm{m}$ in length and $20-200 \mathrm{~nm}$ in diameter. Their composition may be varied in the process of growth, which makes it possible to fabricate embedded quantum dots (QDs) [1].

These objects have a number of unique properties. First, since the size of a QD is precisely controlled, the positions of energy levels in it are also well-defined [2]. Second, several dots may be positioned in a single wire with rather accurately controlled intervals between them. This provides an opportunity to create chains of two or more QDs with a predefined tunnel interaction between neighboring dots. Third, one is free to produce ordered NW arrays. Finally, since a wire itself is a macroscopic object, it is possible to fabricate contacts for current pumping of a single QD. This makes the system very promising for both fundamental and practical research.

\section{EXPERIMENTAL}

AlGaAs nanowires containing GaAs quantum dots were studied. These structures were grown on semiinsulating GaAs [111] substrates in an EP1203 MBE system fitted with a solid $\mathrm{Ga}$ and $\mathrm{Al}$ source and additional arsenic cells. The standard catalytic technique of NW growth was used [3]. The direction of NW growth is exactly perpendicular to the surface of substrates with the indicted orientation.

The samples were characterized using scanning and transmission electron microscopes (SEM and TEM). The synthesized NWs were as long as 500$1000 \mathrm{~nm}$ and $20-50 \mathrm{~nm}$ in diameter; the QD thickness was $2-5 \mathrm{~nm}$.

Photoluminescence (PL) spectra were measured under continuous and pulsed excitation at temperatures ranging from 5 to $250 \mathrm{~K}$ (depending on the intensity of optical excitation and an external magnetic field). A Jobin-Yvon $50 \mathrm{~cm}$ monochromator and a CCD detector were used in these measurements. Microphotoluminescence spectra of an isolated NW were measured with a microscope. The minimum size of a laser spot on the sample was $\sim 1.5 \mu \mathrm{m}$, which made it possible to isolate the signal from a single NW at a nanowire density of $10^{-11} \mathrm{~cm}^{-2}$. Isolated $\mathrm{Al}_{0.26} \mathrm{Ga}_{0.74} \mathrm{As}$ NWs $20-25 \mathrm{~nm}$ in diameter and $1 \mu \mathrm{m}$ in length with a QD grown inside were examined. The thickness of this QD was $2.5-3.0 \mathrm{~nm}$, and its diameter was $15-20 \mathrm{~nm}$.

Figure 1 presents the PL spectrum of an isolated NW under very weak optical excitation (below $\left.0.1 \mathrm{~W} / \mathrm{cm}^{2}\right)$. A narrow (0.2-meV-wide) symmetric line associated with the recombination of an exciton in an isolated QD embedded into a nanowire is seen. As the photoexcitation intensity increased, this line shifted in jumps to one and/or another side. The rate of these jumps increased with the excitation intensity. As a 


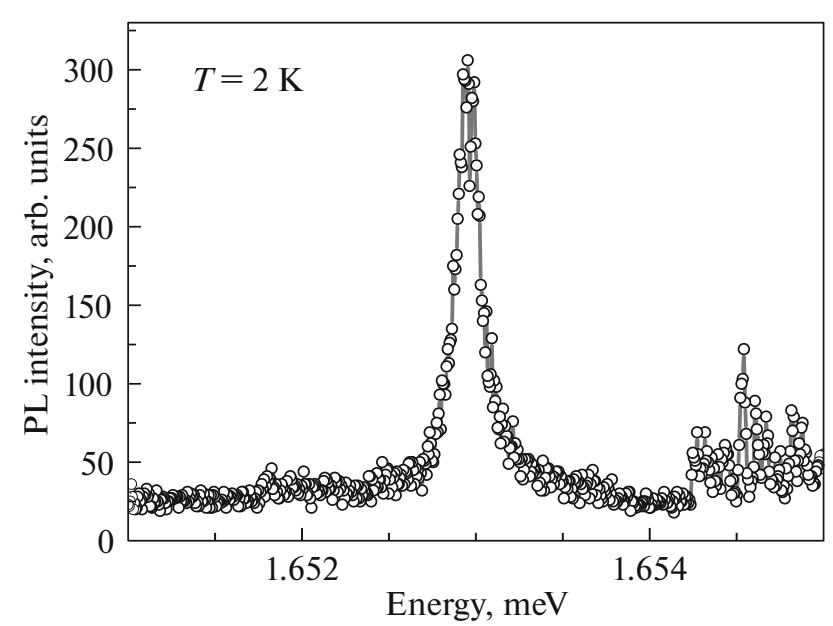

Fig. 1. Exciton PL spectrum from an isolated GaAs quantum dot embedded into an $\mathrm{Al}_{0.26} \mathrm{Ga}_{0.74}$ As quantum wire. The QD thickness and diameter are 2.5 and $15 \mathrm{~nm}$, respectively.

result, it was detected by the photodetector as a very broad line with a width of $1-2 \mathrm{meV}$.

Since an isolated nanoobject was studied, inhomogeneous line broadening is out of the question. The emergence of a broad exciton PL line under sufficiently intense photoexcitation was attributed to the effect of dynamic broadening [4]. The position of the PL line in an NW with a small diameter (i.e., with an exciton being very close to the surface) depends on the presence of charges on the NW surface. Under relatively intense optical excitation, the charge distribution on the NW surface changes. These changes manifest themselves as jumplike line shifts.

PL spectra were measured in magnetic fields up to $8 \mathrm{~T}$ in order to determine the exciton parameters. Figure 2 presents the field dependence of the energy shift of the exciton luminescence line in right-hand $\left(\sigma^{+}\right)$ and left-hand $\left(\sigma^{-}\right)$circular polarizations.

These dependences were used to determine the effective $g$-factor and the diamagnetic shift constant of an exciton. The former was estimated at 1.0 [5], and the latter turned out to be considerably smaller than the diamagnetic shift of an exciton in bulk GaAs [6]. The obtained small shift is indicative of a high exciton binding energy in a QD embedded into a nanowire.

The dependence shown in Fig. 2 yields a value of $d \approx 3 \times 10^{-3} \mathrm{meV} / \mathrm{T}^{2}$ for the diamagnetic shift constant of an exciton in a quantum dot embedded into a nanowire. It is known that the diamagnetic shift constant is related to the exciton radius in the following way [7]:

$$
d=\frac{e^{2}}{8 \mu c^{2}} a_{\mathrm{B}}^{2}
$$

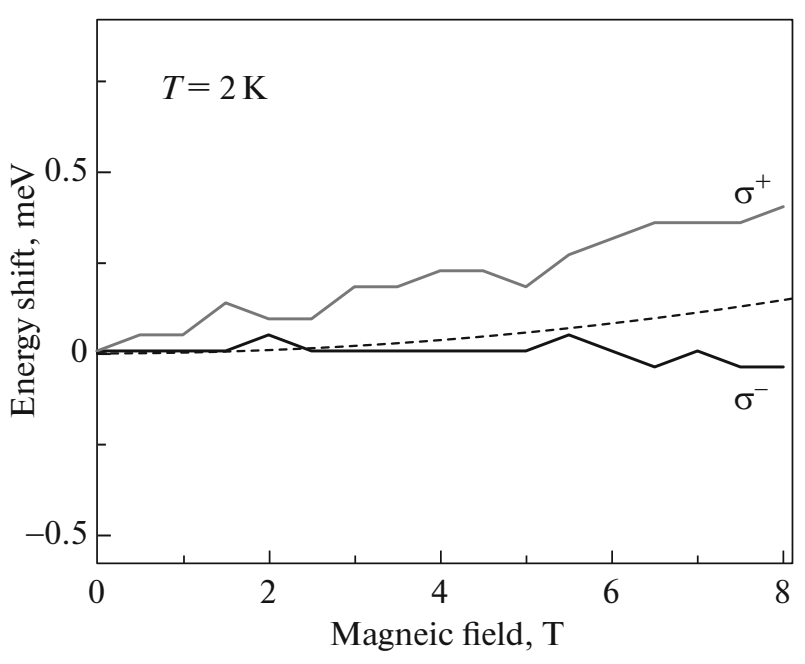

Fig. 2. Field dependence of the energy shift of lines of exciton PL from the sample with an NW containing a quantum dot. The magnetic field strength varies from 0 to $8 \mathrm{~T}$. The dashed curve represents an approximation of the half-sum of two Zeeman components.

where $\mu$ is the reduced mass, $c$ is the speed of light, $e$ is the electron charge, and $a_{\mathrm{B}}$ is the Bohr radius of an exciton in the plane perpendicular to the magnetic field.

The diamagnetic shift constant in wide GaAs quantum wells with a width of $40 \mathrm{~nm}$ (essentially a bulk material) is $0.06 \mathrm{meV} / \mathrm{T}^{2}$ [6]. The diamagnetic shift constant of a bulk exciton may also be estimated by correcting the measured diamagnetic shift in bulk CdTe [8] for the difference in effective masses. This also yields a value close to $0.06 \mathrm{meV} / \mathrm{T}^{2}$. Thus, knowing the Bohr radius of an exciton in bulk GaAs, we find that the Bohr radius of an exciton in the plane perpendicular to the wire axis is $2.8 \mathrm{~nm}$, which is approximately 4.5 times smaller than its bulk value and considerably smaller than the wire radius.

If an exciton in our NW is spherically symmetric, a binding energy of $90 \mathrm{meV}$ corresponds to the Bohr radius indicated above.

It was demonstrated in [9-11] that the binding energy of an exciton in an NW may be as large as several hundred millielectronvolts or even several electronvolts. This increase is induced by the enhancement of Coulomb interaction in an exciton due to dielectric contrast: the static permittivity of the wire material is $\sim 13$, and the permittivity of the surrounding medium is 1 . Therefore, charges within a wire are affected by forces from their image potential, which tend to enhance their confinement. In addition, the screening of Coulomb interaction in an exciton within an NW is weaker [9-11]. 
The full band offset in our NWs is $\sim 300 \mathrm{meV}$. The formula for the exciton PL line position

$$
E_{\mathrm{PL}}=E_{g}^{\mathrm{GaAs}}+E_{c}+E_{v}-E_{\mathrm{exc}},
$$

where $E_{c}$ and $E_{V}$ are the size quantization energies of an electron and a hole and $E_{\text {exc }}$ is the exciton binding energy, may then be used to estimate the size quantization energies of carriers. At $E_{\mathrm{PL}}=1.653 \mathrm{meV}$ and $E_{\text {exc }}=90 \mathrm{meV}$, the total quantization energy of carriers should be $224 \mathrm{meV}$. This energy corresponds to carriers confined in a quantum dot with a thickness of $2.5-3.0 \mathrm{~nm}$ that is surrounded by barriers with an overall height of $300 \mathrm{meV}$ along the axis and by infinitely high in-plane barriers.

Thus, it follows that an exciton is more spherical than quasi-two-dimensional in form. A qualitatively similar result is obtained for an exciton binding energy of $50 \mathrm{meV}$.

An intriguing effect was observed in the polarized photoluminescence spectra of an isolated nanowire. It was found that exciton emission in the [111] direction is polarized linearly in axes [1 $\overline{1} 0]$ and perpendicular axis $[11 \overline{2}]$. These directions coincide with the corresponding crystallographic axes of the substrate. Degree $P_{\text {lin }}$ of linear polarization

$$
P_{\text {lin }}=\frac{I_{[1 \overline{1}]}-I_{[11 \overline{2}]}}{I_{[11 \overline{0}]}+I_{[11 \overline{2}]}}
$$

varied within the $10-50 \%$ range from one sample to the other.

Figure 3 shows the polarized PL spectra of an exciton from an isolated QD within an NW.

It is evident that exciton emission along the wire axis (direction [111]) is linearly polarized. This is surprising, since the growth axis of wires corresponds to the crystallographic three-fold axis with high symmetry (at least $C_{3}$ for both possible crystalline phases: wurtzite and sphalerite). Therefore, linear polarization is definitely not related to NW anisotropy in the plane perpendicular to axis [111]. Two important factors need to be mentioned: (1) the polarization degree varies strongly from one sample to the other; (2) the polarization direction is tied to crystallographic axes of the substrate. The detected luminescence line is specific in that its polarization is not associated with line splitting, which was often the case in anisotropic quantum dots [12].

It is evident that linear polarization is associated with the anisotropy of optical properties induced by the reduction of NW symmetry. A polarization degree of $50 \%$ requires equally strong $\mathrm{NW}$ deformation. Detailed TEM and SEM studied reveal that the NW shape is hexagonal (or near-hexagonal). However, deformation in the direction perpendicular to the NW axis is still a feasible reason for the observed linear polarization of exciton emission along the [111] axis.

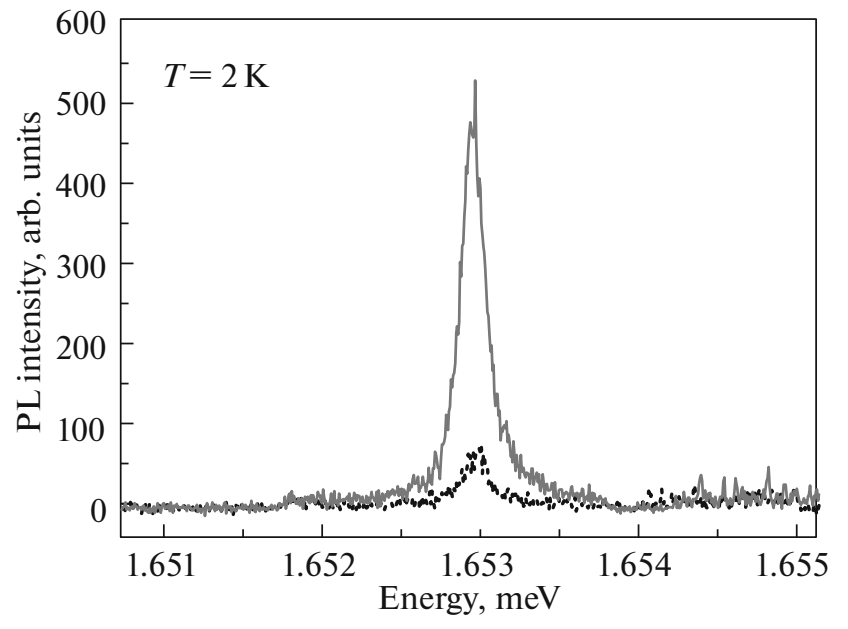

Fig. 3. Linearly polarized PL spectrum. Solid and dashed curves represent polarization along [1 $\overline{1} 0]$ and [11 $\overline{2}]$, respectively.

The only other reason we can suggest is nonaxial positioning of a quantum dot within a nanowire.

The lack of PL line splitting into components polarized along axes $[11 \overline{0}]$ and $[11 \overline{2}]$ suggests that the anisotropy of distribution of the electromagnetic field around an NW (and not the anisotropy of exciton wave functions) is the primary cause of linear PL polarization. The NW shape anisotropy inevitably leads to the anisotropy of exciton wave functions and, consequently, to level splitting. Therefore, in our view, nonaxial positioning of a QD within an NW is a more plausible explanation of the observed linear polarization.

The results of experiments with a magnetic field demonstrate that the dielectric contrast may exert a considerable influence on an exciton. Therefore, even a slight perturbation of the electromagnetic field induced by a QD shift from the NW center may also have a significant effect. According to our estimates, a $10 \%$ shift of the QD center may induce linear polarization with a degree of $\sim 30 \%$.

Optical experiments alone do not allow one to determine the cause of nonaxial QD positioning. However, this is likely to be associated with differences between growth rates in different crystallographic directions. This anisotropy may also be induced by the presence of a mixed wurtzite-sphalerite phase in NWs [3] or by inclined positioning of sources in the MBE chamber. Further studies with electron microscopes are needed in order to determine the causes.

The spectra of polarized photoluminescence of a "resting" nanowire were also studied. In these experiments, a single nanowire was "cut" from the substrate and positioned on a special silicon template.

The PL spectra of a "resting" NW were recorded using a microscope and a spectrometer fitted with a 

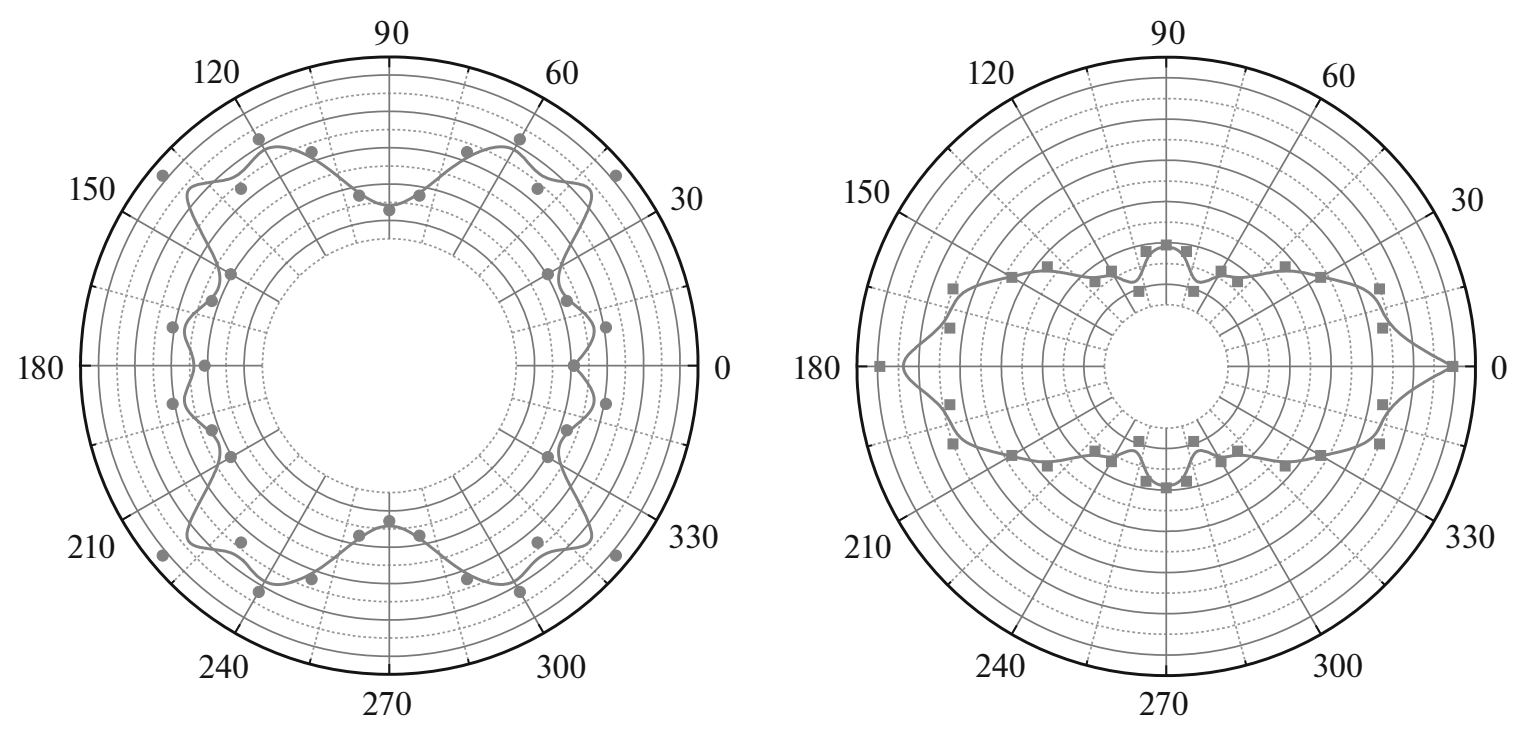

Fig. 4. Diagram of linear polarization of emission from a quantum dot in a "resting" quantum wire.

CCD detector. The spatial resolution of the experimental setup was $\sim 1.5 \mu \mathrm{m}$. Since the studied NW was very small in comparison with the wavelength of light and the spatial resolution, it looked like a bright dot in the optical microscope. Therefore, it was not possible to associate the polarization direction with the nanowire axis. The pattern of polarization in all directions was measured (see Fig. 4).

It can be seen that the exciton emission in a QD from the side of an NW does not feature pronounced linear polarization. If the exciton in the examined structure was quasi-two-dimensional (i.e., its diameter was larger than the thickness along the NW axis), the exciton-transition selection rules would make its emission completely polarized linearly in the direction perpendicular to the wire axis [13]. The emission component polarized along the wire axis should be lacking in the case of exciton-heavy hole annihilation. However, all the observed lines are either weakly polarized or unpolarized.

This suggests that the exciton state with a light hole is the ground one in the studied QD. A light exciton has dipole-moment components both codirectional with and perpendicular to the wire axis.

The geometrical ratio of the QD thickness to its diameter is as large as 10 . Therefore, its properties should be similar to those of a quantum well. However, the exciton state with a light hole becomes the ground one due to additional dielectric confinement in the plane perpendicular to the wire and to the insufficient height of barriers at the dot-wire boundary along the wire axis [14]. As a result, the emission from the NW side is unpolarized. The presence of weak linear polarization (or complete lack of such polarization) is associated with the NW parameters (specifically, its diameter). It seems that the emission from the side face is polarized linearly in NWs with large diameters and is unpolarized in small-diameter NWs. This is related to the form of the wave function of an exciton: if it is localized inside a QD like a quasi-twodimensional one, polarization is observed; if a quantum dot is just a weak localizing potential and the excition is quasi-one-dimensional, polarization is lacking.

\section{CONCLUSIONS}

Two intriguing effects were observed in the spectra of polarized photoluminescence of an isolated quantum dot embedded into a nanowire. Considerable linear polarization (up to 50\%) was detected in recombination of an exciton in the ground state, where this polarization was entirely unexpected and was not observed in earlier experiments [15]. Conversely, very weak linear polarization (or lack of polarization) was noted in cases where complete linear polarization was expected and observed in [15].

The first effect (unexpected detection of polarization) is attributed to the anisotropy of optical properties caused either by nanowire deformation or by nonaixal positioning of a quantum dot within a wire. In the context of strong dielectric contrast, anisotropic dielectric enhancement of the exciton-light interaction may induce marked anisotropy in the plane perpendicular to the wire axis.

The second effect is the detection of unexpectedly weak linear PL polarization (or complete lack of polarization). This is attributed to the fact that the exciton state with a light hole becomes the ground one in a QD with additional dielectric confinement perpendicular to the wire axis. 


\section{ACKNOWLEDGMENTS}

V.P. Kochereshko acknowledges support from the "Electron Correlations in Systems with Strong Interaction" program of the Physical Sciences Division of the Russian Academy of Sciences. The work of A.V. Platonov was supported by program no. 9 "Terahertz Optoelectronics and Spintronics" of the Presidium of the Russian Academy of Sciences.

\section{REFERENCES}

1. V. N. Kats, V. P. Kochereshko, A. V. Platonov, T. V. Chizhova, G. E. Cirlin, A. D. Bouravleuv, Yu. B. Samsonenko, I. P. Soshnikov, E. V. Ubyivovk, J. Bleuse, and H. Mariette, Semicond. Sci. Technol. 27, 015009 (2012).

2. D. Barettin, A. V. Platonov, A. Pecchia, V. N. Kats, G. E. Cirlin, A. D. Bouravleuv, L. Besombes, H. Mariette, M. Auf Der Maur, and A. di Carlo, IEEE J. Sel. Top. Quantum Electron. 19, 1901209 (2013).

3. V. G. Dubrovskii, Theoretical Principles of Technology of Semiconductor Nanostructures (SPb. Gos. Univ., St. Petersburg, 2006) [in Russian].

4. A. V. Platonov, V. P. Kochereshko, V. N. Kats, G. E. Tsyrlin, A. D. Buravlev, Yu. B. Samsonenko, L. Besombes, and H. Mariette, J. Surf. Invest.: X-ray, Synchrotron Neutron Tech. 7, 622 (2013).

5. M. J. Snelling, E. Blackwood, C. J. McDonagh, R. T. Harley, and C. T. B. Foxon, Phys. Rev. B 45, 3922(R) (1992).
6. D. C. Reynolds, Phys. Rev. B 35, 4515 (1987).

7. L. D. Landau and E. M. Lifshitz, Course of Theoretical Physics, Vol. 3: Quantum Mechanics: Non-Relativistic Theory (Nauka, Moscow, 1974, 3th ed.; Pergamon, New York, 1977, 3rd ed.).

8. L. C. Smith, J. J. Davies, D. Wolverson, S. Gramplin, R. T. Cox, J. Cibert, H. Mariette, V. P. Kochereshko, M. Wiater, G. Karczewski, and T. Wojtowicz, Phys. Rev. B 78, 085204 (2008).

9. L. V. Keldysh, JETP Lett. 29, 658 (1979).

10. E. A. Andryushin and A. P. Silin, Semiconductors 27, 695 (1993).

11. E. A. Mulyarov and S. G. Tikhodeev, J. Exp. Theor. Phys. 84, 151 (1997).

12. D. Gammon, E. S. Snow, B. V. Shanabrook, D. S. Katzer, and D. Park, Phys. Rev. Lett. 76, 3005 (1996).

13. M. H. M. van Weert, N. Akopian, F. Kelkensberg, U. Perinetti, M. P. van Kouwen, J. Gómez Rivas, M. T. Borgström, R. E. Algra, M. A. Verheijen, E. P. A. M. Bakkers, L. P. Kouwenhiven, and V. Zwiller, arXiv:0808.2908 [cond-mat.mes-hall].

14. M. A. Semina and R. A. Suris, Semiconductors 45, 917 (2011).

15. G. Bulgarini, M. E. Reimer, and V. Zwiller, Appl. Phes. Lett. 101, 111112 (2012).

Translated by D. Safin 